See discussions, stats, and author profiles for this publication at: https://www.researchgate.net/publication/336066890

\title{
Identifying the Key Risk Factors for Adverse Psychological Outcomes Among Police Officers A Systematic Literature Review
}

Article in Journal of Traumatic Stress · September 2019

DOI: $10.1002 /$ its.22431

\section{CITATIONS}

7 authors, including:

Larissa Sherwood

Trinity College Dublin

3 PUBLICATIONS 14 CITATIONS

SEE PROFILE

20. Frédérique Vallières

Trinity College Dublin

83 PUBLICATIONS 589 CITATIONS

SEE PROFILE
READS

492

Siobhan Hegarty

University College London

1 PUBLICATION 9 CITATIONS

SEE PROFILE

2. Philip Hyland

National University of Ireland, Maynooth

216 PUBLICATIONS 2,949 CITATIONS

SEE PROFILE

Some of the authors of this publication are also working on these related projects:

Project $\quad$ CONTEXT (THE COLLABORATIVE NETWORK FOR TRAINING AND EXCELLENCE IN PSYCHOTRAUMATOLOGY) View project

Project http://kps.org.pl/en View project 


\title{
Identifying the Key Risk Factors for Adverse Psychological Outcomes Among Police Officers A Systematic Literature Review
}

\author{
Larissa Sherwood (iD ${ }_{1}^{1}$ Siobhan Hegarty, ${ }_{1}^{1}$ Frédérique Vallières (iD,${ }^{1}$ Philip Hyland iD,${ }^{2}$ Jamie Murphy, ${ }^{3}$ \\ Geraldine Fitzgerald, ${ }^{4}$ and Tracey Reid ${ }^{5}$ \\ ${ }^{1}$ Centre for Global Health, School of Psychology, Trinity College Dublin \\ ${ }^{2}$ School of Psychology, Maynooth University \\ ${ }^{3}$ Psychology Research Institute, School of Psychology, Ulster University \\ ${ }^{4}$ The Library of Trinity College Dublin, School of Education \\ ${ }^{5}$ Collaborative Network for Training and Excellence in Psychotraumatology
}

\begin{abstract}
It is well established that police work is highly stressful; however, a paucity of evidence limits our knowledge and understanding of the factors that may lead to, compromise, and/or compound psychological distress for this unique population. To address this lack, the current systematic review was conducted to reveal the individual, organizational, and operational risk factors that have been identified in the research literature to date and their associations with depression, anxiety, posttraumatic stress disorder (PTSD), and burnout. We performed searches on literature published between January 2008 and January 2018 and identified a total of 20 studies that met our inclusion criteria. The results indicated that individual factors, including high levels of neuroticism, low social support, and engaging in passive or avoidant coping strategies, were associated with adverse psychological outcomes. This review identified low social support from colleagues as the most common risk factor linked to adverse psychological outcomes. Other organizational risk factors associated with such outcomes included high work demands, low resources, and low reward. This article demonstrates that individual, organizational, and operational factors all impact the negative psychological outcomes of police officers to some degree. In particular, the current review suggests that certain negative psychological outcomes are likely a result of a combination of risk factors across the three domains of risk, highlighting the necessity to observe psychological risk of police in a more comprehensive way.
\end{abstract}

It is well stablished that law enforcement is a high-risk and high-stress occupation associated with an elevated risk of trauma exposure (Andersen \& Papazoglou, 2015; Colwell, Lyons, Bruce, Garner, \& Miller, 2011; Garbarino, Cuomo, Chiorri, \& Magnavita, 2013). As a result, police officers report a high incidence of work-related mental health problems (Stansfeld, Head, Rasul, Singleton, \& Lee, 2003). In the United Kingdom, research suggests that as many as $90 \%$ of police personnel have experienced stress and poor mental health at work, and when compared to the general working population, police personnel are twice as likely to identify problems with work as the source of their mental health problems (Mind, 2015).

Funding has been provided for the COllabortive Network for Training and Excellence in psychoTraumatology (CONTEXT) program from the European Union's Horizon 2020, under the Marie Sklodowska-Curie Grant Agreement (722523).

Correspondence concerning this article should be addressed to Larissa Sherwood, Centre for Global Health, School of Psychology, Trinity College Dublin. E-mail: sherwola@tcd.ie

(c) 2019 International Society for Traumatic Stress Studies. View this article online at wileyonlinelibrary.com

DOI: $10.1002 /$ jts.22431
The sources of stress associated with police work have primarily been explored across two separate domains: organizational and operational. Organizational stressors are defined as strains associated with working for one's organization and include factors such as budget cuts, heavy workload, and nonstandard working hours (Deschênes, Desjardins, Dussault, \& Walla, 2018; Ma et al., 2015). Operational stressors are specific to the unit or specialist role of the officer and include various stressors such as anticipatory stress (i.e., stress endured while anticipating the call or arrival to a potentially traumatic situation), working in isolation, or being involved in a critical incident (Anderson, Litzenberger, \& Plecas, 2002; Regehr, LeBlanc, Barath, Balch, \& Birze, 2013). Additionally, the individual stressors an officer faces, including things such as a history of childhood trauma, bereavement of a close relative, or financial difficulties, also appear to play an important role in mental health outcomes (Asmundson \& Stapleton, 2008; Chopko \& Schwartz, 2013; Follette, Polusly, \& Milbeck, 1994; Habersaat, Geiger, Abdellaoui, \& Wolf, 2015). To better understand the variation in mental health status of police officers, it is necessary to recognize organizational, operational, and individual stressors. 
Most, if not all, employees will work in an environment subject to organizational stress at some point in their careers. This stress can lead to negative mental health outcomes and poor overall well-being (Spielberger, Vagg, \& Wasala, 2003). In the general workforce, psychosocial problems at work, such as job strain, lack of social support, effort-reward imbalance, and reduced control have all been found to predict the onset of mental health problems (Netterstrom et al., 2008; Stephen \& Bridget, 2006). As an organization, the police force places particular importance on solidarity and socialization, as reflected in the hierarchical nature of police organizations (Sklansky, 2007; Workman-Stark, 2017). Among police, the bravado of the service can act as an additional form of organizational stress, which is similar in other organizations such as the military (Andersen \& Papazoglou, 2014; Deschênes et al., 2018). This can lead to negative workplace behaviors, such as bullying, which has emerged as an important psychosocial hazard with the potential to negatively impact police officers' mental health (Tuckey, Dollard, Saebel, \& Berry, 2010). The stigma associated with mental health problems suffered by police personnel is believed to be a result of the bravado and masculine culture of police work (Workman-Stark, 2017). Specific organizational stressors reported by police in the United Kingdom include organizational change, excessive workload, and pressure from management (Mind, 2015).

Unlike organizational stressors, however, the operational stressors associated with working as a police officer tend to be unique to the profession. Police, among other types of emergency personnel, are exposed to potentially traumatizing events or materials on a frequent, if not daily, basis. As a result, high rates of traumatic exposure and higher rates of trauma-related psychopathology have been observed among police officers as compared to the general population (Maia et al., 2007; Marmar et al., 1999; McCaslin et al., 2006). Examples of traumatic exposures that officers routinely encounter as a part of their work include fatal motor vehicle accidents, child abuse cases, homicide scenes, and being the target of assault (Colwell et al., 2011; McCaslin et al., 2006; Weiss et al., 2010). Specific police roles are associated with additional and unique sources of stress. For example, officers who work in Internet child exploitation units are frequently exposed to indecent images of children (Tomyn, Powell, Cassematis, Smallbone, \& Wortley, 2015), and emergency dispatchers often feel that they are unable to directly assist the individual on the telephone, leading to feelings of powerlessness and isolation (Regehr et al., 2013). As such, the stressors that officers feel vary greatly based on the operational aspects of their role (Habersaat et al., 2015).

Police officers are susceptible to the same individual factors that increase risk of depression, anxiety, posttraumatic stress disorder (PTSD), and burnout among the general population. The extant literature suggests these individual risks include being female, having a prior or family history of psychopathology (Inslicht et al., 2010), personality type, life stress, adverse childhood experiences, and negative coping styles (Carlson \& Dalenberg, 2000; de Graaf, Bijl, Ravelli, Smit, \& Vollebergh,
2002; De Venter, Demyttenaere, \& Bruffaerts, 2013; Javidi \& Yadollahihe, 2012; Schnurr \& Vielhauer, 2000; Spector \& O'Connell, 1994; Yuan et al., 2011) Although the sources of individual stress among the general population have been studied in depth, the way in which these individual stressors interact with the stress unique to police work are not well understood.

Taken together, the existing literature suggests that adverse mental health consequences for police officers are most likely due to a combination of risk factors across three domains: individual, organizational, and operational (Habersaat et al., 2015). To date, however, there has been an overwhelming lack of research addressing the various types of stress, with many articles focusing on one or two areas (Habersaat et al., 2015; Hall, Dollard, Tuckey, Winefield, \& Thompson, 2010). There remains a dearth of evidence regarding the associations between individual, organizational, and operational risk factors and mental health outcomes when studied simultaneously. Previous studies have shown that the most common mental health problems for police personnel include anxiety, depression, and PTSD (Austin-Ketch et al., 2012). More recently, burnout has been examined within the police service, and it appears to be highly relevant to this population, particularly for individuals in high-risk police roles (Aryasri \& Mouly, 2017; Asmundson \& Stapleton, 2008). Previous reviews have focused on individual mental health outcomes and single domains of stress; to date, however, there has not been a review inclusive of the various mental health outcomes and domains of stress. To address this gap, the current review aimed to systematically investigate the relevant literature surrounding the various domains of risk and their associations with the most common adverse mental health outcomes studied: depression, anxiety, PTSD, and burnout.

\section{Method}

\section{Search Strategy}

Systematic searches were conducted across six medical, psychological, and social science databases: PsycINFO, CINHAL, MEDLINE, SCOPUS, EMBASE and Social Sciences Index. A comprehensive list of search headings was created and included three categories: police, psychological outcomes, and risk factors (see Supplementary Table S1). Searches included free text search as well as the appropriate advanced search options in each database. The strategy for this review was to focus solely on articles containing the search terms in the title, abstract, or as a keyword or major heading. The final search strings for each database included a comprehensive list of both free text and advanced search options across the three categories.

\section{Inclusion Criteria}

Studies were included if explicit risk factors of adverse mental health outcomes were identified in the study, the population comprised police officers, and the study included 


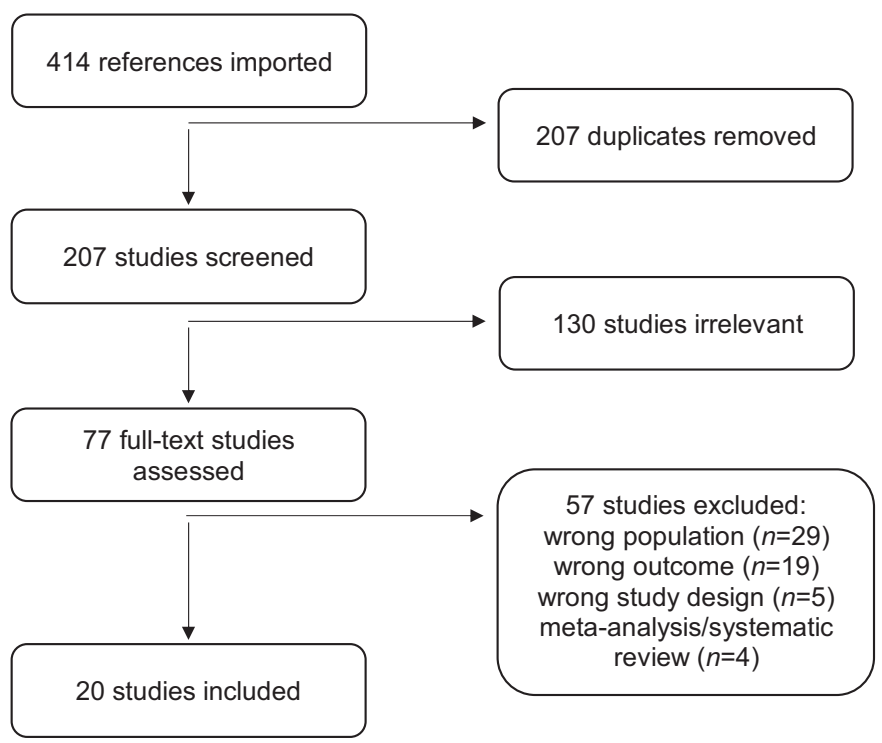

Figure 1. PRISMA flowchart.

psychological measures used to assess one or more of the following psychological states: anxiety, depression, posttraumatic stress, or burnout. Included studies also had to be peer-reviewed journal articles published within the last 10 years (i.e., January 1, 2008-January 1, 2018) for which the abstract and full text were available in English.

\section{Exclusion Criteria}

Studies in which the sample had been working as police personnel for 1 year or less were excluded from this study on the basis that these studies were not comparable to the other included studies. In their first year on the job, officers often spend a majority of their time in training and learn from an experienced field training officer; the first year is commonly a probation period (Tyler \& McKenzie, 2011). Mixed populations of first responders were also excluded, as were studies conducted as a response to a specific event (i.e., terrorism and/or natural disaster), as they are not comparable with the general policing studies in this review.

\section{Selection Process}

Two reviewers agreed on the search terms and assessment criteria for the screening of title, abstract, and full-text articles. The selection process was based on the PRISMA guidelines and was conducted using the systematic review software Covidence (Babineau, 2014; Moher, Liberati, Tetzlaff, \& Altman, 2009). The first literature search produced a total of 1,038 articles, which was reduced to 414 once limits were applied. After screening the articles for title eligibility and removing all duplicate articles across the various databases, a total of 207 articles remained for abstract screening. Figure 1 shows that of the 207 studies, 130 were not relevant based on their abstracts, which left 77 articles for full-text screening. Of the eligible studies for full-text screening, a further 57 articles were excluded, with the primary reasons for exclusion being poor study design, incorrect population, and incorrect outcomes. This resulted in a total of 20 peer-reviewed journal articles for review, which both reviewers agreed on including. Quality assessment of the articles was done using an adapted version of the STROBE and QUALSYST checklists. Once the articles were selected, they were grouped according to various psychological outcomes, and the individual, organizational, and operational risk factors for each outcome were analyzed.

\section{Results}

Of the 20 articles included in this review, $85.0 \%(n=17)$ were cross-sectional and three were prospective, longitudinal designs. All but two articles included both male and female participants. A total of 6,160 participants were included across the 20 studies (Table 1). The reviewed studies covered a diverse range of police forces globally, with one-third of the studies conducted in the United States, one-third conducted across countries in Europe, and the remaining one-third from other parts of the world.

Of the 20 articles included in this review, $35.0 \%(n=7)$ included measures of depression, $15.0 \%(n=3)$ included measures of anxiety, $60.0 \%(n=12)$ included measures of PTSD, and 35\% $(n=7)$ included measures of burnout. Several of the articles included risk factors for multiple outcome measures of adverse mental health. Each of the adverse mental health outcomes and their relevant risk factors were analyzed according to each of the three domains of stress (individual, organizational, and operational) and are outlined in Table 2.

\section{Depression}

Several individual and organizational risk factors were found to be associated with depression. Gender was associated with depression in various studies. For officers working as child abuse investigators, women were found to be more than twice as likely to experience depression compared to their male colleagues (Tehrani, 2016). Violanti (2011) identified physical health as a risk factor associated with depression and found that men and women with higher body mass index (BMI) scores also had higher scores on measures of depression. The personality traits of neuroticism and introversion were found to be associated with depression (Garbarino et al., 2013). Andrew et al. (2013) found that neuroticism and introversion were positively associated with depressive symptoms as were conscientiousness and lack of agreeableness. Sleep quality was shown to be strongly associated with depression, with findings indicating an inverse association between sleep quality and depressive symptoms (Slaven et al., 2011).

Social support plays a significant role in predicting depression in police. Officers who were of lower social standing in their community and among their friends, who also reported less support from superiors and increased loneliness, were at 
Table 1

Article Overviews

\begin{tabular}{|c|c|c|c|c|c|}
\hline Author, Year & Study Design & $n$ & $\begin{array}{l}\text { Participant } \\
\text { information }\end{array}$ & $\begin{array}{l}\text { Mental Health } \\
\text { Measures Used }\end{array}$ & Risk Factor Measured Used \\
\hline Andrew et al., 2013 & Cross-sectional & 412 & $\begin{array}{l}\text { Buffalo, NY, United } \\
\quad \text { States }\end{array}$ & $\begin{array}{l}\text { Dep: CESD } \\
\text { PTSD: IES } \\
\text { Anx: BAI }\end{array}$ & $\begin{array}{l}\text { Pers: Bartone Scale, Brief } \\
\text { COPE, NEO Five-Factor } \\
\text { Inventory, Cook-Medley } \\
\text { Hostility Scale }\end{array}$ \\
\hline Brady, 2016 & Cross-sectional & 443 & ICAC, United States & BO: ProQOL-V & $\begin{array}{l}\text { Pers: Self-Care Practices } \\
\text { Questionnaire } \\
\text { Org: Work-related } \\
\text { factors-developed measure } \\
\text { Oper: Work-related } \\
\text { factors-developed measure }\end{array}$ \\
\hline $\begin{array}{l}\text { De la Fuente } \\
\text { Solana, } 2013\end{array}$ & Cross-sectional & 747 & $\begin{array}{l}\text { National police, } \\
\text { Spain }\end{array}$ & BO: MBI & $\begin{array}{l}\text { Pers: NEO Five-Factor } \\
\quad \text { Inventory }\end{array}$ \\
\hline $\begin{array}{l}\text { Ellrich \& Baier, } \\
2017\end{array}$ & Cross-sectional & 681 & $\begin{array}{l}\text { Assaulted police, } \\
\text { Germany }\end{array}$ & PTSD: PSS & $\begin{array}{l}\text { Pers, Org, Op: Pre-, peri- and } \\
\text { posttraumatic } \\
\text { factors-developed measure }\end{array}$ \\
\hline Fyhn et al., 2016 & Cross-sectional & 156 & $\begin{array}{l}\text { Police investigators, } \\
\text { Norway }\end{array}$ & BO: MBI & $\begin{array}{l}\text { Pers: Dispositional Resilience } \\
\text { Scale 15-R, developed } \\
\text { measure, Subjective Health } \\
\text { Complaints } \\
\text { Org: Job Content } \\
\text { Questionnaire, Utrecht } \\
\text { Work Engagement Scale }\end{array}$ \\
\hline $\begin{array}{l}\text { Garbarino et al., } \\
2013\end{array}$ & Cross-sectional & 289 & $\begin{array}{l}\text { Special forces unit, } \\
\text { Italy }\end{array}$ & $\begin{array}{l}\text { Dep: BDI } \\
\text { Anx: STAI } \\
\text { BO: MBI }\end{array}$ & $\begin{array}{l}\text { Pers: Big Five Questionnaire } \\
\text { Org: Demand, Control, } \\
\text { Support Questionnaire; ERI } \\
\text { Questionnaire }^{\text {b }}\end{array}$ \\
\hline $\begin{array}{l}\text { Habersaat et al., } \\
\qquad 2015\end{array}$ & Cross-sectional & 84 & $\begin{array}{l}\text { Criminal, } \\
\text { emergency, and } \\
\text { community } \\
\text { divisions, } \\
\text { Switzerland }\end{array}$ & $\begin{array}{l}\text { Dep: BDI } \\
\text { PTSD: Mississippi } \\
\text { Scale-Police } \\
\text { Version }\end{array}$ & $\begin{array}{l}\text { Pers: MacArthur Scales of } \\
\text { Subjective Social Status, } \\
\text { Social Readjustment Rating } \\
\text { Scale, French Ways of } \\
\text { Coping Checklist, UCLA } \\
\text { Loneliness Scale, } \\
\text { Cohen-Hoberman Inventory } \\
\text { of Physical Symptoms } \\
\text { Org: Job Content } \\
\text { Questionnaire, French } \\
\text { Perceived Stress Scale at } \\
\text { Work scale }\end{array}$ \\
\hline Hartley et al., 2013 & Cross-sectional & 359 & $\begin{array}{l}\text { Buffalo, NY, United } \\
\quad \text { States }\end{array}$ & $\begin{array}{l}\text { PTSD: } \\
\text { PCL-Civilian } \\
\text { Version }\end{array}$ & Oper: Police Incident Survey \\
\hline $\begin{array}{c}\text { Komarovskaya } \\
\text { et al., } 2011\end{array}$ & Longitudinal & & $\begin{array}{l}n=400, \text { Recruited } \\
\text { at academy, } \\
\text { United States }\end{array}$ & $\begin{array}{l}\text { Dep: BDI-II } \\
\text { PTSD: Mississippi } \\
\text { Scale-Civilian } \\
\text { Version }\end{array}$ & $\begin{array}{l}\text { Pers: Michigan Alcohol } \\
\text { Screening Test } \\
\text { Org: SAS-Self } \\
\text { Oper: Critical Incident } \\
\text { History Questionnaire }\end{array}$ \\
\hline
\end{tabular}


Table 1

Continued

\begin{tabular}{|c|c|c|c|c|c|}
\hline Author, Year & Study Design & $n$ & $\begin{array}{l}\text { Participant } \\
\text { information }\end{array}$ & $\begin{array}{l}\text { Mental Health } \\
\text { Measures Used }\end{array}$ & Risk Factor Measured Used \\
\hline Kula, 2017 & Cross-sectional & 538 & $\begin{array}{l}\text { National police, } \\
\text { Turkey }\end{array}$ & $\begin{array}{l}\text { BO: Copenhagen } \\
\text { Burnout } \\
\text { Inventory }\end{array}$ & $\begin{array}{l}\text { Org: Organizational Police Stress } \\
\text { Questionnaire, Job Satisfaction } \\
\text { Survey } \\
\text { Oper: Operational Police Stress } \\
\text { Questionnaire }\end{array}$ \\
\hline $\begin{array}{l}\text { Kuma \& } \\
\text { Kamalanabhan, } \\
2017\end{array}$ & Cross-sectional & 491 & $\begin{array}{l}\text { Inspector or } \\
\text { subinspector } \\
\text { rank, India }\end{array}$ & BO: MBI & $\begin{array}{l}\text { Org: developed measure, Perceived } \\
\text { Workload Scale (adapted), } \\
\text { Organizational Justice Scale } \\
\text { (adapted), }{ }^{\mathrm{b}} \text { Interpersonal Justice } \\
\text { Scale (adapted) }\end{array}$ \\
\hline Lee et al., 2016 & Cross-sectional & 112 & $\begin{array}{l}\text { Men aged } \geq 50 \\
\text { years } \\
\text { South Korea }\end{array}$ & $\begin{array}{l}\text { Dep: The Korean } \\
\text { Center for } \\
\text { Epidemiologic } \\
\text { Studies- } \\
\text { Depression } \\
\text { Scale } \\
\text { PTSD: IES } \\
\text {-Revised } \\
\text { (Korean ver.) }\end{array}$ & $\begin{array}{l}\text { Pers: Connor-Davidson Resilience } \\
\text { Scale (Korean version) } \\
\text { Org: Korean Occupational Stress } \\
\text { Scale-Short Form } \\
\text { Oper: Self-Report critical incident } \\
\text { exposure (developed by research } \\
\text { team) }\end{array}$ \\
\hline Maia et al., 2011 & Cross-sectional & 212 & Men, Brazil & PTSD: PCL-C & $\begin{array}{l}\text { Pers: Peritraumatic Dissociative } \\
\text { Experiences Questionnaire, } \\
\text { Peritraumatic Stress Inventory, } \\
\text { Sources of Support, Positive and } \\
\text { Negative Affectivity Schedule } \\
\text { Oper: Critical Incident History } \\
\text { Questionnaire }\end{array}$ \\
\hline $\begin{array}{l}\text { Marchand et al., } \\
2015\end{array}$ & Longitudinal & 83 & $\begin{array}{l}\text { Police in major } \\
\text { cities, Canada }\end{array}$ & $\begin{array}{l}\text { Dep: BDI-II } \\
\text { PTSD: Modified } \\
\text { PSS, SCID-I }\end{array}$ & $\begin{array}{l}\text { Pers: Sociodemographic and } \\
\text { work-related variables (developed } \\
\text { by authors), LEC, Perceived } \\
\text { Social Support, Coping Inventory } \\
\text { for Stressful Events, Self-Efficacy } \\
\text { Scale, Short Hardiness Scale, } \\
\text { Initial Subjective Reaction Scale } \\
\text { of Potential Stressful Events } \\
\text { Interview (subscale), } \\
\text { Peritraumatic Dissociative } \\
\text { Experiences Questionnaire } \\
\text { Org: Sociodemographic and } \\
\text { work-related variables (developed } \\
\text { by authors) } \\
\text { Oper: Sociodemographic and } \\
\text { work-related variables (developed } \\
\text { by authors), TSQ }\end{array}$ \\
\hline
\end{tabular}

(Continued) 
Table 1

Continued

\begin{tabular}{|c|c|c|c|c|c|}
\hline Author, Year & Study Design & $n$ & $\begin{array}{l}\text { Participant } \\
\text { information }\end{array}$ & $\begin{array}{l}\text { Mental Health } \\
\text { Measures Used }\end{array}$ & Risk Factor Measured Used \\
\hline $\begin{array}{l}\text { Martin, Marchand, } \\
\text { \& Boyer, } 2009\end{array}$ & Cross-sectional & 132 & $\begin{array}{l}\text { Montreal area, } \\
\text { Canada }\end{array}$ & PTSD: SCID-I & $\begin{array}{l}\text { Pers: LEC, History of } \\
\text { familial psychopathology, } \\
\text { WAS, Short Hardiness } \\
\text { Scale, Coping Inventory for } \\
\text { Stressful Situations, } \\
\text { Peritraumatic Dissociative } \\
\text { Experiences } \\
\text { Questionnaire-Self-Report, } \\
\text { Potential Stressful Events } \\
\text { Interview, Perceived Social } \\
\text { Support Questionnaire } \\
\text { Org: Organizational support } \\
\text { (developed measure) } \\
\text { Oper: Trauma severity } \\
\text { (developed measure) }\end{array}$ \\
\hline $\begin{array}{l}\text { Martin, Marchand, } \\
\text { Boyer, \& Martin, } \\
2009\end{array}$ & Cross-sectional & 169 & Montreal, Canada & PTSD: SCID-I & $\begin{array}{l}\text { Pers: LEC, Short Hardiness } \\
\text { Scale, Coping Inventory } \\
\text { for Stressful Situations }\end{array}$ \\
\hline Slaven et al., 2011 & Cross-sectional & 391 & $\begin{array}{l}\text { Buffalo, NY, United } \\
\quad \text { States }\end{array}$ & Dep: CESD & $\begin{array}{l}\text { Pers: Pittsburgh Sleep } \\
\quad \text { Quality Index }\end{array}$ \\
\hline Tehrani, 2016 & Cross-sectional & 126 & $\begin{array}{l}\text { Child abuse, United } \\
\text { Kingdom }\end{array}$ & $\begin{array}{l}\text { Anx, Dep, BO: QoL } \\
\text { PTSD: } \\
\text { IES-Extended }\end{array}$ & $\begin{array}{l}\text { Pers: Eysenck Personality } \\
\text { Questionnaire (short form) }\end{array}$ \\
\hline Violanti, 2011 & Cross-sectional & 99 & United States & Dep: CESD & $\begin{array}{l}\text { Pers: BMI, abdominal height, } \\
\text { and waist circumference }\end{array}$ \\
\hline Yuan et al., 2011 & Longitudinal & 233 & $\begin{array}{l}\text { Recruited at } \\
\text { academy, United } \\
\text { States }\end{array}$ & $\begin{array}{l}\text { PTSD: Mississippi } \\
\text { Scale-Civilian } \\
\text { Anx: SCID-I }\end{array}$ & $\begin{array}{l}\text { Pers: Life Stressor } \\
\text { Checklist-Revised, NEO } \\
\text { Five Factor Inventory, } \\
\text { World Assumptions Scale, } \\
\text { Sources of Social Support } \\
\text { Scale, Social Adjustment } \\
\text { Scale-Self Report } \\
\text { Oper: Critical Incident } \\
\text { History Questionnaire }\end{array}$ \\
\hline
\end{tabular}

Note $:$ Dep = depression; Anx = anxiety; PTSD = posttraumatic stress disorder; $\mathrm{BO}=$ burnout; Pers = measure of personal risk factor; Org = measure of organizational risk factor; Oper $=$ measure of operational risk factor; CESD = Center for Epidemiological Studies Depression Scale; IES $=$ Impact of Events Scale; BAI = Beck Anxiety Index; ProQOL = Professional Quality of Life scale; PSS = Posttraumatic Stress Disorder Symptom Scale; MBI = Maslach's Burnout Inventory; BDI = Beck Depression Inventory; STAI = State-Trait Anxiety Inventory; PCL = PTSD checklist; SAS = Social Adjustment Scale; SCID-I = Structured Clinical Interview for DSM-IV Axis I Disorders; LEC = Life Events Checklist; TSQ = Trauma Severity Questionnaire; WAS = World Assumptions Scale, QoL = Quality of Life scale, $\mathrm{NEO}=$ Neuroticism-Extraversion-Openness, ERI $=$ Effort Reward Imbalance Questionnaire; ICAC $=$ Internet Crimes Against Children.

a higher risk of depressive symptoms (Habersaat et al., 2015). Andrew et al. (2013) and Habersaat et al. (2015) both found that certain types of coping (problem-focused coping, passive coping, and avoidance) were associated with depressive symptoms. Two of the included studies (Komarovskaya et al., 2011; Violanti et al., 2011) identified relationship status as a significant predictor of depression, with higher depression scores among single or divorced officers.
Organizational factors such as reduced ability to make decisions, lower reward opportunities, higher levels of commitment, more job demands, and poorer relationships with colleagues were all found to be associated with increased depressive symptomatology. Garbarino et al. (2013) found a positive association between length of employment and depression. Organizational commitment was inversely associated with depression, and perceived control in the workplace was significantly associated 
Table 2

Identified Risk Factors

\begin{tabular}{|c|c|c|c|}
\hline & Individual & Organizational & Operational \\
\hline Depression & $\begin{array}{l}\text { Female sex; higher BMI } \\
\text { score; single or divorced; } \\
\text { neuroticism, introversion, } \\
\text { conscientiousness, and lack } \\
\text { of agreeableness; poor } \\
\text { sleep quality; lack of social } \\
\text { support; loneliness; } \\
\text { problem focused coping, } \\
\text { passive coping, and } \\
\text { avoidance }\end{array}$ & $\begin{array}{l}\text { Reduced decision making; } \\
\text { lower reward } \\
\text { opportunities; higher job } \\
\text { demand; perceived control; } \\
\text { poor relationships with } \\
\text { colleagues; length of } \\
\text { employment }\end{array}$ & $\begin{array}{l}\text { No measures of operational } \\
\text { risk }\end{array}$ \\
\hline Anxiety & $\begin{array}{l}\text { Female sex; low emotional } \\
\text { stability; introversion, } \\
\text { neuroticism, lack of } \\
\text { agreeableness }\end{array}$ & $\begin{array}{l}\text { Low social support at work; } \\
\text { high effort and low reward; } \\
\text { lack of commitment }\end{array}$ & $\begin{array}{l}\text { No measures of operational } \\
\text { risk }\end{array}$ \\
\hline PTSD & $\begin{array}{l}\text { Female sex; lack of } \\
\text { agreeableness, } \\
\text { introversion, neuroticism, } \\
\text { and conscientiousness; } \\
\text { negative affect; } \\
\text { comorbidity of depression; } \\
\text { low self-reliance, } \\
\text { self-resilience, and } \\
\text { self-worth; low } \\
\text { benevolence; passive } \\
\text { coping, emotional coping, } \\
\text { and avoidance; lack of } \\
\text { social support; being single } \\
\text { or divorced }\end{array}$ & $\begin{array}{l}\text { Lack of control; commitment; } \\
\text { higher job stress; lack of } \\
\text { preparatory and follow-up } \\
\text { sessions; high workload; } \\
\text { job duration }\end{array}$ & $\begin{array}{l}\text { Increased exposure to } \\
\text { traumatic events; being } \\
\text { ambushed or intentionally } \\
\text { injured while on duty; } \\
\text { direct life threat; killing } \\
\text { someone in the line of duty }\end{array}$ \\
\hline Burnout & $\begin{array}{l}\text { Having a romantic partner; } \\
\text { neuroticism, extraversion, } \\
\text { agreeableness, openness, } \\
\text { and conscientiousness; } \\
\text { negative coping } \\
\text { techniques; lack of social } \\
\text { support }\end{array}$ & $\begin{array}{l}\text { High demand, high effort and } \\
\text { low reward; } \\
\text { overcommitment; } \\
\text { inflexible working hours; } \\
\text { perceived unfairness; } \\
\text { work-family conflict; role } \\
\text { ambiguity; work overload; } \\
\text { low organizational, } \\
\text { colleague, and supervisor } \\
\text { support; feeling frequently } \\
\text { overwhelmed }\end{array}$ & $\begin{array}{l}\text { Working as an agent; frequent } \\
\text { exposure to crimes against } \\
\text { children }\end{array}$ \\
\hline
\end{tabular}

Note. $\mathrm{PTSD}=$ posttraumatic stress disorder; $\mathrm{BMI}=$ body mass index.

with depression (Andrew et al., 2013). No articles assessed operational risk factors as a risk factor for depression.

\section{Anxiety}

The three articles that included measures of anxiety also included measures of depression, and many of the risk factors associated with depression were also associated with anxiety. Individual risk factors for anxiety included low emotional stability, introversion, and less agreeableness, and organizational risk factors included low levels of support, low reward, and higher effort (Garbarino et al., 2013). Neuroticism significantly predicted anxiety (Tehrani, 2016). Andrew et al. (2013) also concluded that neuroticism, introversion, being disagreeable, and conscientiousness were significantly associated with symptoms of anxiety.

Cognitive restructuring and active coping were inversely associated with anxiety (Andrew et al., 2013). The hardiness 
factor of "challenge," whereby officers see challenges as opportunities to grow, was significantly associated with anxiety for men. Commitment (i.e., finding purpose and meaning in stressful events) was inversely associated with anxiety for both men and women. Similar to what was found regarding depression, female officers working as Internet child abuse investigators (ICAC) were more than twice as likely as their male colleagues to experience anxiety (Tehrani, 2016). The included articles dealing with anxiety primarily focused on individual risk factors, particularly personality, and some areas of organizational risk. There were no operational risk factors found in the included articles.

\section{Posttraumatic Stress Disorder}

The results of the search yielded the highest number of articles for PTSD. A total of 12 articles assessed the various risk factors for PTSD in police officers, and these articles included all three domains of risk (individual, organizational, and operational). Regarding the individual risk domain, we identified sex, number of children, personality type, coping mechanisms, and social support as risk factors impacting outcomes of PTSD. A majority of the studies included sex differences for risk factors. One study on specialist ICAC officers found that being female predicted PTSD (Tehrani, 2016). Another study (Marchand, Boyer, Nadeau, Beaulieu-Prevost, \& Martin, 2015) identified that number of children was positively associated with more severe symptoms but only for men with more than one child.

There were some differences between male and female officers with regards to personality type as a risk factor for PTSD. Agreeableness and extraversion were found to be inversely associated with PTSD symptoms in both sexes (Andrew et al., 2013). In addition, extraversion was identified as being negatively correlated with PTSD for both men and women officers (Tehrani, 2016). Conscientiousness was significantly associated with PTSD symptoms but only among male officers (Andrew et al., 2013). Neuroticism was identified as a significant predictor of PTSD in three studies (Andrew et al., 2013; Tehrani, 2016; Violanti, 2011). For women, openness was associated with PTSD symptomology, and the sex interaction was significant (Andrew et al., 2013). Negative affect was shown to be significantly and positively associated with PTSD symptom severity (Maia et al., 2011). Two studies (Maia et al., 2011; Martin, Marchand, Boyer, \& Martin, 2009) measured peritraumatic dissociation and found it to be a very strong predictor of PTSD for police personnel involved in a critical incident. More specifically, the impact of dissociation on PTSD symptoms was intensified for individuals with higher levels of negative affect (Maia et al., 2011).

One study identified depression, lower self-reliance, and low levels of self-resilience as strong predictors of PTSD symptoms (Lee et al., 2016). Another study found that officers who were experiencing full PTSD were 4 times more likely to have depressive disorder (60\% vs. $15 \%$ ) as compared to officers who did not have a diagnosis of PTSD ((Martin, Marchand, \& Boyer,
2009). Additionally, higher ratings of self-worth and assumptions of a benevolent world were associated with lower levels of PTSD symptomology for police officers (Yuan et al., 2011).

Two studies included assessment of the personality trait of "hardiness." The first found that the hardiness dimension "challenge" was associated with PTSD symptoms for men but not women (Andrew et al., 2013), whereas the second found that challenge was associated with full and partial PTSD symptoms (Martin, Marchand, Boyer et al., 2009). Conflicting results were found for both the "control" and "commitment" components of hardiness. Andrew et al. (2013) identified that control and commitment were inversely associated with PTSD symptomology, whereas Martin, Marchand, Boyer et al. (2009) found no association.

Several articles included coping strategies as a dimension of individual risk. Passive coping and avoidance were associated with PTSD symptoms, with the association for women significantly stronger than for men, $p=.03$ (Andrew et al., 2013). It was identified that the more officers resorted to emotional coping strategies to deal with stress, the more likely they were to develop symptoms of PTSD (Marchand et al., 2015). Social support appeared across the literature as a crucial protective factor against the development of PTSD and other adverse mental health outcomes. For example, lack of social support and being single or divorced was found to be a significant predictor of PTSD (Komarovskaya et al., 2011), whereas higher levels of social support were associated with lower levels of PTSD symptoms (Maia et al., 2011; Martin, Marchand, \& Boyer, 2009; Martin, Marchand, Boyer et al., 2009; Yuan et al., 2011).

Although most of the articles that were included in the PTSD group discussed various individual risk factors, many articles also touched on organizational and operational stress. We identified a small number of articles that included organizational risk factors for the development of PTSD. One study of male officers found that a higher level of job stress was linked to higher levels of PTSD symptoms (Lee et al., 2016). In a study that looked at high-risk and low-risk clusters of officers, the authors found that community division officers in the highrisk category were at an increased risk of posttraumatic stress symptoms (Habersaat et al., 2015). The high-risk cluster was characterized by lower social status, decision latitude, support, and problem-focused coping as well as higher ratings of loneliness. In contrast, regular preparatory and follow-up sessions-a crucial area of training and debriefing for policing-were found to reduce posttraumatic stress (Ellrich \& Baier, 2017).

The operational risk factors included for PTSD covered both general operational risk of police as well as specific critical incidents. Several studies (Hartley, Sarkisian, Violanti, Andrew, \& Burchfiel, 2013; Maia et al., 2011; Yuan et al., 2011) identified the number of traumatic events or frequency of critical incident exposure as significant predictors for the development of PTSD among police. For early-career police officers, less cumulative critical incident exposure over the first 2 years of service was correlated with lower levels of PTSD symptoms (Yuan et al., 2011). For female officers, particularly those with a large 
workload, a positive relation was found between frequency of traumatic events and PTSD symptoms (Hartley et al., 2013). However, the same study identified female officers who had no prior trauma exposure and had witnessed "abused children" or "homicide victims" to be at an increased risk of PTSD symptomatology compared to officers with prior trauma exposure.

Job duration was found to significantly predict PTSD symptom severity (Maia et al., 2011). A detailed study on PTSD severity found that officers with partial PTSD had significantly more years of experience than officers without PTSD (Martin, Marchand, Boyer et al., 2009). Additionally, in comparison to officers without PTSD, officers with partial PTSD were significantly more likely to also have substance-related disorders $(22 \%$ vs. $1 \%)$ and major depressive disorder (44\% vs. $15 \%)$. However, no significant differences were found for the full PTSD group.

The current review included three studies that accounted for specific critical incidents to which officers may be exposed. First, a study on officers after they were victims of violent assaults found that being ambushed, taking more time off work, and being intentionally injured while on duty reported increased posttraumatic stress symptoms (Ellrich \& Baier, 2017). Second, one study identified that female officers who had been involved in a shooting had higher PTSD symptom scores (Hartley et al., 2013). Third, a study reported that exposure to direct life threat was found to be a significant predictor of PTSD (Komarovskaya et al., 2011); this study also found that killing or injuring someone in the line of duty was associated with PTSD symptoms.

\section{Burnout}

Several of the seven studies on burnout included individual risk factors, and two included operational risks. Due to the nature of burnout, most of the risk factors for burnout analyzed by these studies focused on organizational risks. Three of the articles (De la Fuente Solana, Aguayo Extremera, Vargas Pecino, \& Cañadas de la Fuente, 2013; Garbarino et al., 2013; Kumar \& Kamalanabhan, 2017) assessed risk factors in relation to the three specific dimensions of Maslach's Burnout Inventory (MBI; emotional exhaustion, depersonalization, and personal accomplishment) individually.

The first dimension MBI examines "emotional exhaustion." Garbarino et al. (2013) found that high demand, effort, and overcommitment as well as low reward were associated with emotional exhaustion. In a second study, inflexible working hours, perceived unfairness, work overload, role ambiguity along with work-home interference, work-family conflict, and familywork conflict were all associated with emotional exhaustion (Kumar \& Kamalanabhan, 2017). A third study (De la Fuente Solana et al., 2013) identified two individual risk factors: (a) police officers who had romantic partners had significantly higher scores on measures of emotional exhaustion than police officers without partners, and (b) the personality variables neuroticism, agreeableness, conscientiousness, and extraversion were all significantly correlated with emotional exhaustion.
"Depersonalization" is the second MBI domain and is defined as the development of negative feelings and attitudes toward both work colleagues and the individuals that officers assist (Maia et al., 2011). Several protective factors for this domain were identified, including having children, friendliness, emotional stability, control, support, and reward (Garbarino et al., 2013). Additionally, overcommitment and effort were both identified as risk factors for burnout. Similar to emotional exhaustion, the organizational risk factors of burnout for depersonalization included perceived unfairness, inflexible work hours, role ambiguity, and work-family conflict (Kumar \& Kamalanabhan, 2017). In contrast, De la Fuente Solana et al. (2013) found no significant differences between work-related variables, except for officers with partners, who had higher burnout scores. De la Fuente Solana and colleagues also found several personality variables that were significant and consistent with emotional exhaustion: neuroticism, agreeableness, conscientiousness, and extraversion.

The final MBI dimension is "personal accomplishment," for which, in contrast to emotional exhaustion and depersonalization, lower scores are associated with higher levels of burnout. Inflexible working hours have been most strongly associated with this component of burnout, followed by family-work conflict, perceived unfairness, work-family conflict, role ambiguity, and work overload (Kumar \& Kamalanabhan, 2017). One study identified that officers between the ages of 45 and 50 years had much lower personal accomplishment scores than those who were 25-30 or 40-45 years of age (De la Fuente Solana et al., 2013). Additionally, police officers without children and without partners reported higher scores as did officers with fewer than 10 years of experience.

Various risk factors were also identified outside of the MBI. Three articles analyzed individual risk factors, including a study of an ICAC taskforce, which found that having a strong support system outside of work and using positive coping techniques mitigated the risk of burnout (Brady, 2016). Another study by Tehrani (2016) conducted on a similar police population found that neuroticism significantly predicted burnout. All five personality factors of the NEO Five-Factor Inventory (NEO-FFI; i.e., neuroticism, extraversion, openness, agreeableness, and conscientiousness) were significantly correlated with burnout. Similarly, another study found that all of the personality variables on the Big Five Questionnaire (conscientiousness, extraversion, friendliness, openness, and emotional stability) positively predicted the personal accomplishment component of burnout (Garbarino et al., 2013). Fyhn, Fjell, and Johnsen (2016) found that hardiness-commitment (i.e., the commitment aspect of the personality style that allows an individual to cope proactively and positively with challenges), social support, marital status, and work engagement were the only variables that significantly predicted burnout.

More than half of the studies in the burnout category identified low organizational support as a risk factor for burnout (Brady, 2016; Fyhn et al., 2016; Garbarino et al., 2013; Kumar \& Kamalanabhan, 2017). Social support, both outside 
of work and at work, was found to be significantly associated with burnout across four of the studies. One study of police investigators (Fyhn et al., 2016) and a similar study on an ICAC unit (Brady, 2016) found that low social support from colleagues and supervisors as well as a low level of organizational support were significantly associated with higher levels of burnout. When looking at a Demand Control Support model, developed by Karasek (1979) to establish a model in which the two main sources of work stress are "demand" and "control" and are moderated by "support," Garbarino et al. (2013) found that support was negatively associated with burnout but solely in the depersonalization domain of the MBI. A similar study by Kumar and Kamalanabhan (2017) found that work support was significantly and negatively associated with burnout across all three MBI domains. In addition to social support in the workplace, frequently feeling overwhelmed, inflexible working hours, perceived unfairness, work overload, role ambiguity, and a low level of work engagement were all associated with an increased risk of burnout (Brady, 2016; Fyhn et al., 2016; Kumar \& Kamalanabhan, 2017).

Although both organizational and operational stress have significant effects on burnout (Kula, 2017), only three articles in this review included measures for operational risk factors. Garbarino et al. (2013) found that officers working as agents were at a higher risk of professional exhaustion (an MBI domain) when compared to supervisors or technical staff. For the ICAC taskforce, frequent indirect exposure to crimes against children was associated with higher rates of burnout (Brady, 2016). The final study identified that organizational stressors had more influence in determining rates of burnout among the police force than did operational stressors (Kula, 2017).

\section{Discussion}

In light of the lack of research investigating how specific individual, organizational, and operational risk factors are associated with adverse mental health outcomes among police officers, this review systematically investigated various adverse psychological outcomes as a result of working as a police officer across these three domains of risk. Although all the articles included assessments of individual risk and more than half assessed organizational risk factors, far fewer included variables on operational risk factors.

Several patterns of individual risk factors emerged across the adverse mental health outcomes for police personnel. These included lack of social support, personality types, negative coping styles, and being female. It is not surprising that these variables recurred in this review as they are common individual risk factors throughout the literature on mental health (Carlson et al., 2016; de Graaf et al., 2002; Javidi \& Yadollahihe, 2012; Schnurr $\&$ Vielhauer, 2000). The personality traits of neuroticism and introversion; negative coping styles, such as passive coping or avoidance; and low levels of social support were found to be consistent risk factors for all adverse mental health outcomes.
Congruent with previous studies (Hart \& Wearing, 1995), neuroticism, introversion, and maladaptive coping styles are wellestablished risk factors for multiple types of negative mental health states.

Lack of social support from colleagues at work as well as from family and friends was positively associated with the mental health outcomes. Consistent with previous findings, being female was found to be a risk factor (Bramness, Walby, Hjellvik, Selmer, \& Tverdal, 2010; Carlson et al., 2016; Fuhrer, Stansfeld, Chemali, \& Shipley, 1999). Some individual stressors that were not accounted for in the articles of this review, such as a history of childhood trauma, bereavement of a close relative, or financial difficulties, have also been shown to play an important role in mental health outcomes (Asmundson \& Stapleton, 2008; Chopko \& Schwartz, 2013; Follette et al., 1994; Habersaat et al., 2015) and should therefore be included in future studies conducted with police officers.

Whereas previous research has focused primarily on the operational stressors associated with policing, recent advances in the field suggest that organizational stress may be just as impactful on mental health, if not more so (Moon \& Jonson, 2012). The most common organizational risk identified in this review was social support from colleagues. Other factors include high levels of effort and low levels of reward, which appear to predict higher levels of anxiety, depression, and burnout; lower ratings of decision latitude and loneliness appear to predict PTSD. Officers suffering from burnout tend to point toward organizational stressors, including work demands and resources, as the source of their problems (Kohan \& Mazmanian, 2016; Martinussen, Richardsen, \& Burke, 2007). The role of organizational stressors in predicting adverse mental health outcomes among police is consistent with work by Toch (2002), who found that top-down management practices and departmental politics were more stressful than the operational stressors routinely encountered by police personnel. This may be because police officers are aware of the risks associated with the occupation, but they are often unprepared for the organizational stressors which may thus more deeply impact their mental health.

Posttraumatic stress disorder appears to be the mental health outcome most strongly associated with operational risks, with the frequency of traumatic events emerging as a significant predictor for PTSD across various studies (Davidson, Hughes, \& Blazer, 1991; Wrenn et al., 2011). However, previous research has also supported the resilience perspective, whereby positive coping strategies for previous traumas can build resilience and act as a protector of PTSD in future traumas (Hartley et al., 2013; Shing, Jayawickreme, \& Waugh, 2016). This area should be further explored within a police context in order to better understand the cumulative impact of traumatic exposure. Certain types of trauma may have a larger impact than others. For example, it was found that when an officer is involved in a violent incident where there is potential for serious harm or direct life threat, such as being ambushed or being involved in a shooting, the risk of PTSD increases, as does the severity of symptoms 
(Ellrich \& Baier, 2017; Hartley et al., 2013; Komarovskaya et al., 2011).

Frequent indirect exposure to crimes against children was the only operational risk factor identified for burnout. Overall, organizational stressors appear to have a much larger influence on rates of burnout than do operational risks. However, it must be noted that minimal operational risk factor variables were included in the studies in this review. Therefore, the organizational stressors may simply be measured more frequently than the operational risk factors of police work. Future consideration should be given to the various sources of operational stress for police as well as to the severity and frequency of traumatic events encountered by police personnel.

There were some limitations to the current review. The measures used to identify adverse mental health outcomes were inconsistent, indicating a need for standardization in this field of study. Moreover, the individual stressor variables used in the articles subject to this review are not exhaustive and certainly do not represent the full spectrum of individual risk factors. The populations used in each study, although all police personnel, range from specific units to the general police force to earlycareer officers recruited during training. It should also be noted that there may be cultural differences in crime rates, types of crimes, and police practices that have not been accounted for; however, the range of countries included does suggest a generalizability to the findings.

Because most of the studies included in this review were cross-sectional in design, causality cannot be established. Furthermore, none of the selected articles for this review included measures of stigma. Stigma has been identified as a significant area of organizational stress for officers facing psychological difficulties. A survey conducted by Mind (2015) revealed that although police officers experience increased rates of mental health problems in comparison to other emergency service personnel (i.e., fire service and ambulance service), they are actually less likely than the general workforce to take time off work as a result. This is likely a product of mental health stigmatization among police officers due to the masculine, "just get on with it" attitude of the organization. Moreover, three in four police personnel stated that their organization did not encourage them to talk about mental health compared to $45 \%$ of the general population (Mind, 2015).

The number of articles included for each of the mental health outcomes reflects the focus of recent research in the area of police mental health, which has mostly been concentrated around PTSD. It is apparent that the individual, organizational, and operational risk factors all impact, to some extent, psychological outcomes for police officers. The psychopathology of police personnel is multidimensional and interactional across domains. To best understand adverse psychological outcomes of police, it is essential to consider the various sources of stress and risk, as outlined in this review. Not only are police exposed to various critical incidents but they are also subject to the stressors of working in a high-stress, bravado culture. The individual adverse mental health outcomes (e.g., anxiety, depression) are associated with the three domains of risk at varying degrees. For example, PTSD appears most frequently associated with the operational risk factors, whereas burnout is most associated with organizational stressors. Overall, social support is strongly associated with the psychological well-being of police officers across all the adverse mental health outcomes included in this review and is therefore identified as a key protective factor to improve resilience among police.

Research on police mental health has often been conducted to include a single domain of risk, as observed in the various articles included in this review. However, it is commonly known and generally accepted that psychological disorders have a multifactorial aetiology. Future research should therefore be inclusive of individual, organizational and operational risks in order to obtain a more accurate and thorough understanding of how these risk factors interact to impact on adverse psychological outcomes of police. Additionally, this systematic review demonstrates the need to investigate the differences in types of risk for the various discrete units within the police service, as type and frequency of traumatic exposure will vary based on each police role.

\section{References}

Andersen, J. P., \& Papazoglou, K. (2014). Friends under fire: Cross-cultural relationships and trauma exposure among police officers. Traumatology, 20, 182-190. https://doi.org/10.1037/h0099403

Andersen, J. P., \& Papazoglou, K. (2015). Police health professionals' knowledge and practices in educating officers about trauma and health. Journal of Law Enforcement, 5(5). https://doi.org/10.1037/e549362014-001

Anderson, G. S., Litzenberger, R., \& Plecas, D. (2002). Physical evidence of police officer stress. Policing: An International Journal of Police Strategies \& Management, 25, 399-420. https://doi.org/10.1108/13639510210429437

Andrew, M. E., Howsare, J. L., Charles, L. E., McCanlies, E. C., Mnatsakanova, A., Hartley, T. A., . . . Violanti, J. M. (2013). Associations between protective factors and psychological distress vary by gender: The Buffalo CardioMetabolic Occupational Police Stress Study. International Journal of Emergency Mental Health, 15, 12.

Aryasri, A. R., \& Mouly, N. C. (2017). Occupational stress and burnout among police constabulary: An analysis. The Journal of Institute of Public Enterprise, 40(3), 17.

Asmundson, G. J., \& Stapleton, J. A. (2008). Associations between dimensions of anxiety sensitivity and PTSD symptom clusters in active-duty police officers. Cognitive Behavior Therapy, 37, 66-75. https://doi.org/ $10.1080 / 16506070801969005$

Austin-Ketch, T. L., Violanti, J., Fekedulegn, D., Andrew, M. E., Burchfield, C. M., \& Hartley, T. A. (2012). Addictions and the criminal justice system, what happens on the other side? Post-traumatic stress symptoms and cortisol measures in a police cohort. Journal of Addictions Nursing, 23, 22-29. https://doi.org/10.3109/10884602.2011.645255

Brady, P. Q. (2016). Crimes against caring: Exploring the risk of secondary traumatic stress, burnout, and compassion satisfaction among child exploitation investigators. Journal of Police and Criminal Psychology, 32, 305-318. https://doi.org/10.1007/s11896-016-9223-8

Bramness, J. G., Walby, F. A., Hjellvik, V., Selmer, R., \& Tverdal, A. (2010). Self-reported mental health and its gender differences as a predictor of suicide in the middle-aged. American Journal of Epidemiology, 172, 160 166. https://doi.org/10.1093/aje/kwq091 
Carlson, E. B., \& Dalenberg. (2000). A conceptual framework for the impact of traumatic experiences (Vol. 1). Thousand Oaks, CA: Sage Publications.

Carlson, E. B., Palmieri, P. A., Field, N. P., Dalenberg, C. J., Macia, K. S., \& Spain, D. A. (2016). Contributions of risk and protective factors to prediction of psychological symptoms after traumatic experiences. Comprehensive Psychiatry, 69, 106-115. https://doi.org/10.1016/j.comppsych.2016.04.022

Chopko, B. A., \& Schwartz, R. C. (2013). The relation between mindfulness and posttraumatic stress symptoms among police officers. Journal of Loss and Trauma, 18, 1-9. https://doi.org/10.1080/15325024.2012.674442

Colwell, L. H., Lyons, P. M., Bruce, A. J., Garner, R. L., \& Miller, R. S. (2011). Police officers' cognitive appraisals for traumatic events: Implications for treatment and training. Applied Psychology in Criminal Justice, 7, 106-132.

Davidson, J. R. T., Hughes, D. L., \& Blazer, D. G. e. a. (1991). Posttraumatic stress disorder in the community: An epidemiological study. Psychological Medicine, 21, 713-721.

de Graaf, R., Bijl, R., Ravelli, A., Smit, F., \& Vollebergh, W. (2002). Predictors of first incidence of DSM-III-R psychiatric disorders in the general population: Findings from the Netherlands Mental Health Survey and Incidence Study. Acta Psychiatr Scand, 106, 303-313. https://doi.org/10.1034/j.1600-0447.2002.01397.x

De la Fuente Solana, E. I., Aguayo Extremera, R., Vargas Pecino, C., \& Cañadas de la Fuente, G. R. (2013). Prevalence and risk factors of burnout syndrome among Spanish police officers. Psicothema, 25, 488-493. https://doi.org/10.7334/psicothema2013.81

De Venter, M., Demyttenaere, K., \& Bruffaerts, R. (2013). The relationship between adverse childhood experiences and mental health in adulthood: A systematic literature review. Tijdschrift Voor Psychiatrie, 55, 259-268.

Deschênes, A.-A., Desjardins, C., Dussault, M., \& Walla, P. (2018). Psychosocial factors linked to the occupational psychological health of police officers: Preliminary study. Cogent Psychology, 5. https://doi.org/ $10.1080 / 23311908.2018 .1426271$

Ellrich, K., \& Baier, D. (2017). Post-traumatic stress symptoms in police officers following violent assaults: A study on general and police-specific risk and protective factors. Journal of Interpersonal Violence, 32, 331-356. https://doi.org/10.1177/0886260515586358

Follette, V. M., Polusly, M. M., \& Milbeck, K. (1994). Mental health and law enforcement professionals: Trauma history, psychological symptoms, and impact of providing services to child sexual abuse survivors. Professional Psychology: Research and Practice, 25, 275-282. https://doi.org/10.1037//0735-7028.25.3.275

Fuhrer, R., Stansfeld, S., Chemali, J., \& Shipley, M. J. (1999). Gender, social relations and mental health: Prospective findings from an occupational cohort (Whitehall II study). Social Science and Medicine, 48, 77-87. https://doi.org/10.1016/s0277-9536(98)00290-1

Fyhn, T., Fjell, K. K., \& Johnsen, B. H. (2016). Resilience factors among police investigators: Hardiness-commitment a unique contributor. Journal of Police and Criminal Psychology, 31, 261-269. https://doi.org/10.1007/s11896-015-9181-6

Garbarino, S., Cuomo, G., Chiorri, C., \& Magnavita, N. (2013). Association of work-related stress with mental health problems in a special police force unit. BMJ Open, 3(7). https://doi.org/10.1136/bmjopen-2013-002791

Habersaat, S. A., Geiger, A. M., Abdellaoui, S., \& Wolf, J. M. (2015). Health in police officers: Role of risk factor clusters and police divisions. Social Science and Medicine, 143, 213-222. https://doi.org/ 10.1016/j.socscimed.2015.08.043

Hall, G. B., Dollard, M. F., Tuckey, M. R., Winefield, A. H., \& Thompson, B. M. (2010). Job demands, work-family conflict, and emotional exhaustion in police officers: A longitudinal test of competing theories. Journal of Occupational \& Organizational Psychology, 83, 237-250. https://doi. org/10.1348/096317908x401723

Hart, P. M., \& Wearing, A. J. (1995). Police stress and well-being: Integrating personality, coping and daily work experiences. Journal of Occupational \& Organizational Psychology, 68, 133-156. https://doi.org/10.1111/j.2044-8325.1995.tb00578.x

Hartley, T. A., Sarkisian, K., Violanti, J. M., Andrew, M. E., \& Burchfiel, C. M. (2013). PTSD symptoms among police officers: Associations with frequency, recency, and types of traumatic events. International Journal of Emergency Mental Health, 15(4), 12.

Inslicht, S. S., McCaslin, S. E., Metzler, T. J., Henn-Haase, C., Hart, S. L., Maguen, S., ... Marmar, C. R. (2010). Family psychiatric history, peritraumatic reactivity, and posttraumatic stress symptoms: A prospective study of police. Journal of Psychiatric Research, 44, 22-31. https://doi.org/10.1016/j.jpsychires.2009.05.011

Javidi, H., \& Yadollahihe, M. (2012). Post-traumatic stress disorder. International Journal of Occupational Environment Medicine, 3(1), 9.

Kohan, A., \& Mazmanian, D. (2016). Police work, burnout, and proorganizational behavior. Criminal Justice and Behavior, 30, 559-583. https://doi.org/10.1177/0093854803254432

Komarovskaya, I., Maguen, S., McCaslin, S. E., Metzler, T. J., Madan, A., Brown, A. D., ... Marmar, C. R. (2011). The impact of killing and injuring others on mental health symptoms among police officers. Journal of Psychiatric Research, 45, 1332-1336. https://doi.org/ 10.1016/j.jpsychires.2011.05.004

Kula, S. (2017). Occupational stress, supervisor support, job satisfaction, and work-related burnout: Perceptions of Turkish National Police (TNP) members. Police Practice \& Research: An International Journal, 18, 146-159. https://doi.org/10.1080/15614263.2016.1250630

Kumar, V., \& Kamalanabhan, T. J. (2017). Moderating role of work support in stressor-burnout relationship: An empirical investigation among police personnel in India. Psychological Studies, 62, 85-97. https://doi.org/10.1007/s12646-017-0383-0

Lee, J. K., Choi, H. G., Kim, J. Y., Nam, J., Kang, H. T., Koh, S. B., \& Oh, S. S. (2016). Self-resilience as a protective factor against development of post-traumatic stress disorder symptoms in police officers. Annals of Occupational and Environmental Medicine, 28, 58-58.

Ma, C. C., Andrew, M. E., Fekedulegn, D., Gu, J. K., Hartley, T. A., Charles, L. E., ... Burchfiel, C. M. (2015). Shift work and occupational stress in police officers. Safety and Health at Work, 6, 25-29. https://doi.org/10.1016/j.shaw.2014.10.001

Maia, D. B., Marmar, C. R., Henn-Haase, C., Nóbrega, A., Fiszman, A., Marques-Portella, C., ... Figueira, I. (2011). Predictors of PTSD symptoms in Brazilian police officers. Brazilian Journal of Psychiatry, 33,5 .

Maia, D. B., Marmar, C. R., Metzler, T., Nobrega, A., Berger, W., Mendlowicz, M. V., . . Figueira, I. (2007). Post-traumatic stress symptoms in an elite unit of Brazilian police officers: Prevalence and impact on psychosocial functioning and on physical and mental health. Journal of Affective Disorders, 97, 241-245. https://doi.org/10.1016/j.jad.2006.06.004

Marchand, A., Boyer, R., Nadeau, C., Beaulieu-Prevost, D., \& Martin, M. (2015). Predictors of posttraumatic stress disorder among police officers: A prospective study. Psychological Trauma: Theory, Research, Practice, and Policy, 7, 212-221. https://doi.org/10.1037/a0038780

Marmar, C. R., Weiss, D. S., Metzler, T. J., Delucchi, K. L., Best, S. R., \& Wentworth, K. A. (1999). Longitudinal course and predictors of continuing distress following critical incident exposure in emergency services personnel. Journal of Nervous and Mental Disease, 187, 15-22. https://doi.org/10.1097/00005053-199901000-00004 
Martin, M., Marchand, A., \& Boyer, R. (2009). Traumatic events in the workplace: Impact on psychopathology and healthcare use of police officers. International Journal of Emergency Mental Health, 11, 165-176.

Martin, M., Marchand, A., Boyer, R., \& Martin, N. (2009). Predictors of the development of posttraumatic stress disorder among police officers. Journal Of Trauma \& Dissociation, 10, 451-468. https://doi.org/ $10.1080 / 15299730903143626$

Martinussen, M., Richardsen, A. M., \& Burke, R. J. (2007). Job demands, job resources, and burnout among police officers. Journal of Criminal Justice, 35, 239-249. https://doi.org/10.1016/j.jcrimjus.2007.03.001

McCaslin, S. E., Rogers, C. E., Metzler, T. J., Best, S. R., Weiss, D. S., Fagan, J. A., ... Marmar, C. R. (2006). The impact of personal threat on police officers' responses to critical incident stressors. Journal of Nervous and Mental Disease, 194, 591-597. https://doi.org/10.1097/01. nmd.0000230641.43013.68

Mind. (2015). Blue Light Scoping Survey. Retrieved from https://www.mind. org.uk/

Moher, D., Liberati, A., Tetzlaff, J., \& Altman, D. G. (2009). Preferred reporting items for systematic reviews and meta-analyzes: The PRISMA statement. PLoS Med, 6(7). https://doi.org/10.1371/journal.pmed1000097

Moon, M. M., \& Jonson, C. L. (2012). The influence of occupational strain on organizational commitment among police: A general strain theory approach. Journal of Criminal Justice, 40, 249-258. https://doi.org/ 10.1016/j.jcrimjus.2012.02.004

Netterstrom, B., Conrad, N., Bech, P., Fink, P., Olsen, O., Rugulies, R., \& Stansfeld, S. (2008). The relation between work-related psychosocial factors and the development of depression. Epidemiologic Reviews, 30, 118-132. https://doi.org/10.1093/epirev/mxn004

Regehr, C., LeBlanc, V. R., Barath, I., Balch, J., \& Birze, A. (2013). Predictors of physiological stress and psychological distress in police communicators. Police Practice and Research, 14, 451-463. https://doi.org/ $10.1080 / 15614263.2012 .736718$

Schnurr, P. P., \& Vielhauer, M. J. (2000). Personality as a risk factor for PTSD. In R. Yehuda (Ed.), Risk factors for posttraumatic stress disorder (pp. 191-222). Washington, DC: American Psychiatric Press.

Shing, E. Z., Jayawickreme, E., \& Waugh, C. E. (2016). Contextual positive coping as a factor contributing to resilience after disasters. Journal of Clinical Psychology, 72, 1287-1306. https://doi.org/10.1002/jclp.22327

Sklansky, D. A. (2007). Seeing blue: Police reform, occupational culture, and cognitive burn-in. Bingley, United Kingdom: Emerald Group Publishing Limited.

Slaven, J. E., Mnatsakanova, A., Burchfiel, C. M., Smith, L. M., Charles, L. E., Andrew, M. E., ... Violanti, J. M. (2011). Association of sleep quality with depression in police officers. International Journal of Emergency Mental Health, 13, 267-277.

Spector, P. A., \& O'Connell, B. J. (1994). The contribution of personality traits, negative affectivity, locus of control and Type A to the subsequent reports of job stressors and job strains. Journal of Occupational \& Organizational Psychology, 67, 1-12. https://doi.org/10.1111/j.2044-8325.1994.tb00545.x
Spielberger, C. D., Vagg, P. R., \& Wasala, C. F. (2003). Occupational stress: Job pressures and lack of support. In J. C. Quick, L. E. Tetrick, J. C. Quick, \& L. E. Tetrick (Eds.), Handbook of occupational health psychology. (pp. 185-200). Washington, DC: American Psychological Association.

Stansfield, S., \& Candy, B. (2006). Psychosocial work environment and mental health - a meta-analytic review. Scandinavian Journal of Work, Environment \& Health, (6), 443-462. https://doi.org/10.5271/sjweh.1050

Stansfeld, S. A., Head, J., Rasul, F., Singleton, N., \& Lee, A. (2003). Occupation and mental health: Secondary analyzes of the ONS Psychiatric Morbidity Survey of Great Britain (168). Retrieved from http://www.hse.gov.uk/research/rrpdf/rr168.pdf

Tehrani, N. (2016). Extraversion, neuroticism and secondary trauma in Internet child abuse investigators. Occupupational Medicine, 66, 403-407. https://doi.org/10.1093/occmed/kqw004

Toch, H. (2002). Stress in policing. Washington, DC: American Psychological Association.

Tomyn, A. J., Powell, M. B., Cassematis, P., Smallbone, S., \& Wortley, R. (2015). Examination of the subjective well-being of Australian Internet child exploitation investigators. Australian Psychologist, 50, 203-11. https://doi.org/10.1111/ap.12119

Tuckey, M. R., Dollard, M. F., Saebel, J., \& Berry, N. M. (2010). Negative workplace behavior: Temporal associations with cardiovascular outcomes and psychological health problems in Australian police. Stress and Health, 26, 372-81. https://doi.org/10.1002/smi.1306

Tyler, M. A., \& McKenzie, W. E. (2011). Mentoring first year police constables: Police mentors' perspectives. Journal of Workplace Learning, 23, 518-530. https://doi.org/10.1108/13665621111174870

Violanti, J. M. (2011). Police organizational stress: The impact of negative discipline. International Journal of Emergency Mental Health, 13, 3136.

Violanti, J. M., Fekedulegn, D., Andrew, M. E., Charles, L. E., Hartley, T. A., \& Burchfiel, C. M. (2011). Adiposity in policing: Mental health consequences. International Journal of Emergency Mental Health, 13, 257-266.

Weiss, D. S., Brunet, A., Best, S. R., Metzler, T. J., Liberman, A., Pole, N., .. Marmar, C. R. (2010). Frequency and severity approaches to indexing exposure to trauma: The Critical Incident History Questionnaire for police officers. Journal of Traumatic Stress, 23, 734-743. https://doi.org/10.1002/jts.20576

Workman-Stark, A. L. (2017). Understanding police culture. Inclusive Policing from the Inside Out (pp. 19-35). Cham, Switzerland: Springer.

Wrenn, G. L., Wingo, A. P., Moore, R., Pelletier, T., Gutman, A. R., Bradley, B., \& Ressler, K. J. (2011). The effect of resilience on posttraumatic stress disorder in trauma-exposed inner-city primary care patients. Journal of the National Medical Association, 103, 560-566. https://doi.org/10.1016/s0027-9684(15)30381-3

Yuan, C., Wang, Z., Inslicht, S. S., McCaslin, S. E., Metzler, T. J., HennHaase, C., ... Marmar, C. R. (2011). Protective factors for posttraumatic stress disorder symptoms in a prospective study of police officers. Psychiatry Research, 188, 45-50. https://doi.org/10.1016/j.psychres.2010.10.034 